\title{
Orthogonally protected 1,2-diols from electron rich alkenes using metal-free olefin syn-dihydroxylation
}

\author{
Ignacio Colomer, ${ }^{\mathrm{a}}$ Rosimeire Coura Barcelos, ${ }^{\mathrm{a}}$ Kirsten E. Christensen ${ }^{\mathrm{a} \S}$ and Timothy J. Donohoe ${ }^{\mathrm{a} *}$ \\ Department of Chemistry, University of Oxford, Chemistry Research Laboratory, Mansfield Road, Oxford, OXI 3TA (UK)
}

\begin{abstract}
A new method for the stereoselective metal-free syn dihydroxylation of electron rich olefins is reported, involving reaction with TEMPO/IBX in trifluoroethanol (TFE) or hexafluoroisopropanol (HFIP), and the addition of a suitable nucleophile. Orthogonally protected syn 1,2-diols were obtained with high levels of diastereocontrol; and these products were selectively deprotected and selectively functionalized into synthetically useful compounds.
\end{abstract}

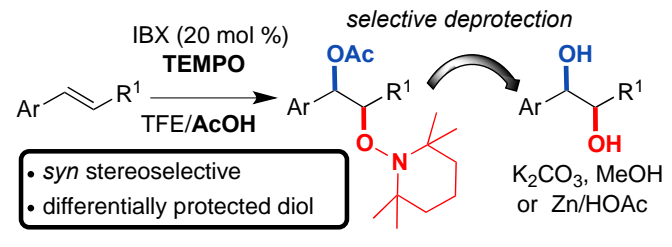

Olefin dihydroxylation is one of the most powerful tools in organic chemistry. The functionalization of vicinal carbons via double $\mathrm{C}-\mathrm{O}$ bond formation can be achieved in a single step and established dihydroxylation methods give high levels of stereospecificity. ${ }^{1}$ In particular, $\mathrm{OsO}_{4}$ has been used extensively in organic synthesis for the syn dihydroxylation of olefins, although its toxic nature is well recognized.

The development of new methods to overcome the use of toxic reagents is of great interest and many groups have been devoted to finding new metal-free dihydroxylation procedures. ${ }^{2}$ Peroxides, ${ }^{3}$ hydroxamic acids ${ }^{4}$ and hypervalent iodine reagents ${ }^{5}$ used in stoichiometric amounts give good yields of syn-dioxygenation products, with modest to good diastereocontrol. Chiral hypervalent iodine reagents have also been used in diacetoxylation ${ }^{6}$ and lactonization reactions. ${ }^{7}$ In addition, the use of sub-stochiometric amounts of hypervalent iodine reagents has been developed recently. ${ }^{8}$ Alternatively the use of stable persistent radicals such as nitroxyl radicals, in particular (2,2,6,6-tetramethylpiperidin-1-yl)oxyl (TEMPO), has allowed a breakthrough in this area. ${ }^{9}$ Although great advances have been made in the functionalization of olefins using TEMPO, with an emphasis on stereoselective intermolecular aminooxygenation, ${ }^{10}$ azidooxygenation, ${ }^{11}$ oxyarylation $^{12}$ and trifluorooxygenation, ${ }^{13}$ the dioxygenation reaction itself remains under-explored. ${ }^{14}$

Following our recent research on the stereoselective synthesis of cyclobutanes II via oxidation of styrenes to a radical cation I, we became intrigued with the idea of trapping this radical cation intermediate (Scheme 1). ${ }^{15}$ The use of TEMPO has led to the discovery of a new metal-free dihydroxylation process with the incorporation of both TEMPO and an external nucleophile (or the solvent in its absence).

Scheme 1. Metal-free dihydroxylation using hypervalent iodine and TEMPO in fluorinated solvents.

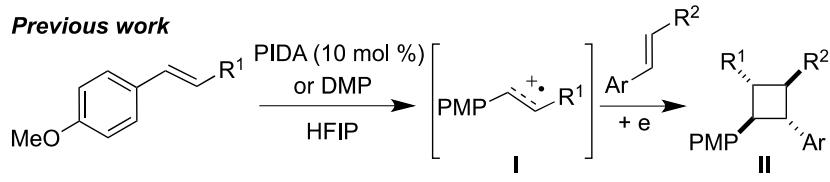

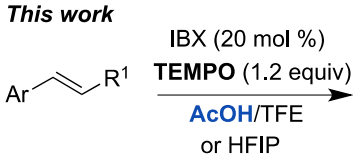

$\mathrm{PMP}=p-\mathrm{MeOC}_{6} \mathrm{H}_{4}$ $\mathrm{PIDA}=\mathrm{Phl}(\mathrm{OAc})_{2}$ $\mathrm{IBX}=2$-lodoxybenzoic acid

$\mathrm{DMP}=$ Dess- Martin periodinane

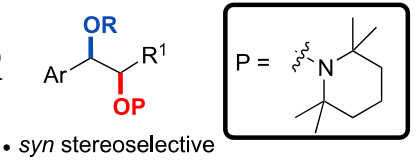

- differentially protected diol

- metal-free dihydroxylation

We began using trans-anethole $\mathbf{1 a}$ as a model alkene under previously optimized conditions for the synthesis of cyclobutanes (10 mol \% of PIDA in hexafluoroisopropanol (HFIP) at $\mathrm{rt}$ ), with the addition of 1.2 equiv of TEMPO as a radical trapping agent (Table 1, entry 1). The protected diol 2a was obtained in $37 \%$ yield as a single syn diastereoisomer. Using hypervalent iodine(V) gave better results, with IBX as the most promising, affording $\mathbf{2 a}$ in $66-76 \%$ yield (Table 1 , entries 2 and 3). Changing to trifluoroethanol (TFE) gave no product with any of the hypervalent iodine reagents used (Table 1, entries 4-6). ${ }^{16}$ Increasing the oxidant loading, using $20 \mathrm{~mol} \%$ of IBX in HFIP, improved the yield up to $85 \%$ with high syn stereocontrol (Table 1, entry 7). Interestingly, the use of an acidic non-fluorinated solvent, such as $\mathrm{AcOH}$ gave the analogous product 3a, but with much lower diastereocontrol (Table 1, entry 8). In sharp contrast when using a polar protic but non-acidic solvent, such as EtOH, no product was observed. Finally, some control experiments were carried on without using any hypervalent iodine additive. While the use of TFE gave no reaction (Table 1, entry 10, in agreement with entries 4-6), surprisingly the reaction in HFIP or AcOH allowed isolation of the corresponding product $\mathbf{2 a / 3 a}$ (Table 1 , entries 11 and 12), in low yield (compare entries 11 vs. 7 and 12 vs. 8). It is also noteworthy that a much better diastereocontrol is again observed with HFIP than with AcOH. Finally taking advantage of the lack of reactivity in TFE (compared to HFIP) 
using a 1:1 mixture of TFE/AcOH gave 3a in 93\% yield and with high diastereocontrol (Table 1 entry 13 vs. entry 8 ).

Table 1. Optimization of the dioxygenation of $1 \mathrm{a}$.

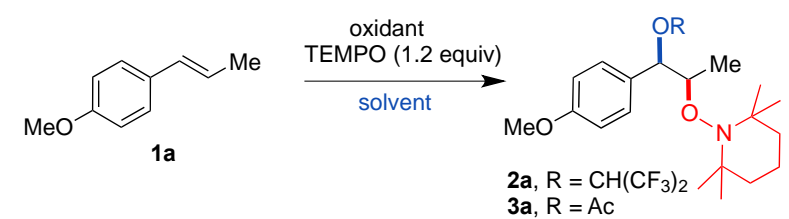

\begin{tabular}{|c|c|c|c|c|}
\hline entry & solvent & oxidant & yield of 2 or $3(\%)$ & $\mathrm{dr}$ \\
\hline 1 & HFIP & $10 \mathrm{~mol} \% \mathrm{PIDA}$ & $2 a(37)$ & $\geq 95: 5$ \\
\hline 2 & HFIP & $10 \mathrm{~mol} \% \mathrm{DMP}$ & $2 a(66)$ & $\geq 95: 5$ \\
\hline 3 & HFIP & $10 \mathrm{~mol} \% \mathrm{IBX}$ & 2a (76) & $\geq 95: 5$ \\
\hline $4^{a}$ & TFE & $10 \mathrm{~mol} \%$ PIDA & 0 & - \\
\hline $5^{a}$ & TFE & $10 \mathrm{~mol} \% \mathrm{DMP}$ & 0 & - \\
\hline $6^{a}$ & TFE & $10 \mathrm{~mol} \% \mathrm{IBX}$ & 0 & - \\
\hline 7 & HFIP & $20 \mathrm{~mol} \% \mathrm{IBX}$ & $\mathbf{2 a}(85)$ & $\geq 95: 5$ \\
\hline 8 & $\mathrm{AcOH}$ & $20 \mathrm{~mol} \% \mathrm{IBX}$ & $3 \mathbf{a}(70)$ & $60: 40$ \\
\hline 9 & $\mathrm{EtOH}$ & $20 \mathrm{~mol} \% \mathrm{IBX}$ & 0 & - \\
\hline 10 & TFE & - & 0 & - \\
\hline $11^{b}$ & HFIP & - & $2 a(45)$ & $\geq 95: 5$ \\
\hline $12^{b}$ & $\mathrm{AcOH}$ & - & $3 \mathbf{a}(42)$ & $60: 40$ \\
\hline 13 & $\mathrm{TFE} / \mathrm{AcOH}$ & $20 \mathrm{~mol} \% \mathrm{IBX}$ & $3 \mathbf{a}(93)$ & $\geq 95: 5$ \\
\hline
\end{tabular}

a traces of the corresponding cyclobutane were observed, however almost all starting material was recovered. ${ }^{b} 50 \%$ of starting material was recovered

Based on the above experiments our mechanistic proposal is shown in Scheme 2. Observing a strong background reaction in the absence of hypervalent iodine reagent (Table 1, entries 11-12), and being aware of the ability of TEMPO to disproportionate in acidic solvents, ${ }^{17}$ we propose the generation of hydroxylamine III and oxoamonium cation IV. ${ }^{18}$ Electron rich olefin 1a may react with oxoamonium cation IV to form benzylic cation intermediate $\mathbf{V},{ }^{19}$ which will then be trapped by a nucleophile (either an external carboxylic acid or HFIP). ${ }^{20}$ The beneficial role of IBX in this reaction may then be to generate oxoamonium cation IV in situ, therefore improving the yield. ${ }^{21}$ The stereochemical outcome of the cation trapping can be rationalized using intermediate $\mathbf{V}$, with the external nucleophile approaching antiperiplanar to the $\mathrm{R}^{1}$ group, in a conformation that minimises allylic strain, thus rendering a net syn dihydroxylation. At this point we do not rule out the possibility of the OTEMP nitrogen atom in the intermediate cation playing a role in directing the nucleophile. The high levels of diastereocontrol displayed when using fluorinated solvents may be related to their larger dielectric constant $(\varepsilon=26$ for TFE and $\varepsilon=18$ for HFIP) compared to AcOH $(\varepsilon=6.2)$, which may influence the conformation and reactivity of a cationic intermediate. ${ }^{22}$ At this point we cannot comment on the role that ion-pairing plays in controlling the cation reactivity and this factor may well differ between the two solvent regimes. Control experiments which involved resubjecting mixtures or single diastereoisomers of $\mathbf{3 a}$ to the reaction conditions in HFIP or AcOH solvents led to no change in product composition, thus ruling out equilibration at the benzylic centre.

Scheme 2. Proposed reaction pathway.

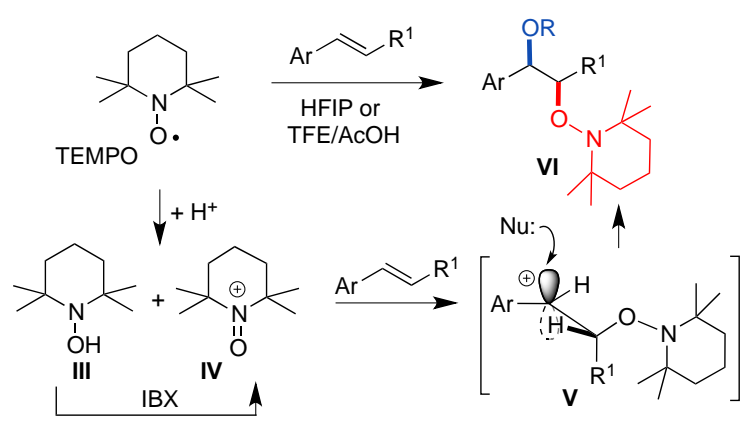

Next, we decided to explore the reaction scope for both the addition of HFIP leading to products 2 (Scheme 3 ) and the incorporation of $\mathrm{AcOH}$ to give orthogonally protected 1,2diols 3 (Scheme 4). The scope for the dioxygenation of alkenes via HFIP addition was explored using styrenes with different electron-rich aromatic rings; therefore starting from $E$-asarone $(\mathrm{Ar}=2,4,5$-trimethoxyphenyl) or from the 3,4dimethoxy derivative, products $\mathbf{2 b}$ and $\mathbf{2 c}$ were obtained. The incorporation of an ortho- $\mathrm{Br}$ substituent in the aromatic ring is tolerated, without diminishing the reactivity (2d) (Scheme 3). Moreover, substituents on the allylic position, such as ethyl, $i$ Pr or cyclopropyl were all compatible (2e-g) (Scheme 3). When ( $Z$ )-1f styrene was used under these conditions, the same syn diastereoisomer $2 \mathbf{f}$ was observed; this stereoconvergence is supporting evidence for the proposed mechanistic pathway proceding via a free cation (see Scheme 2). Note that the regiochemistry and syn relative stereochemistry was proved based on an X-Ray crystal structure of $\mathbf{2 c},^{23}$ and the remaining products in Scheme 3 were assigned by analogy.

Scheme 3. Scope of the metal-free dioxygenation with hexafluoroisopropanol incorporation.

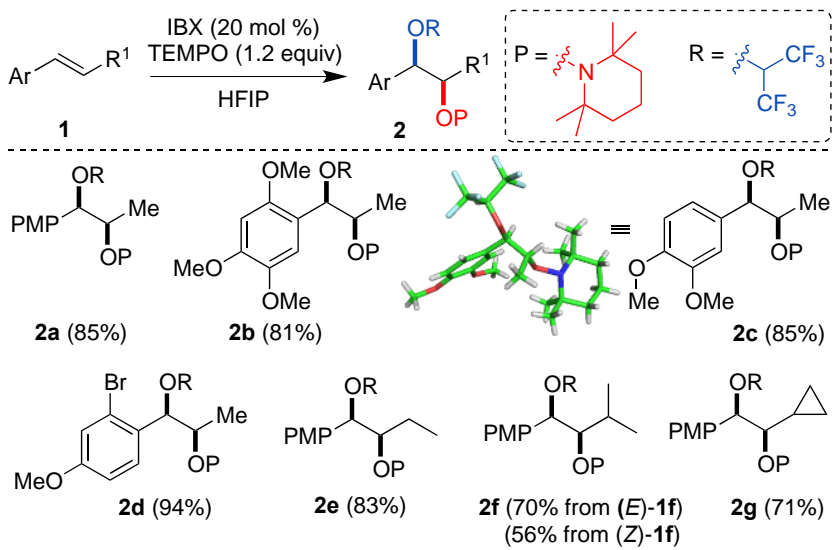

We then focused our efforts on studying the scope of carboxylic acid incorporation because of the easy and selective deprotection protocols that exist. We also decided to concentrate on 1,2-disubstituted alkenes in order to study the diastereocontrol of the process. Keeping the $p$-methoxyphenyl ring (PMP) constant we first investigated the compatibility of the allylic substitution. Aliphatic linear (3b), branched (3c) (Scheme 4) or alicyclic (3d) substitution resulted in good yields of the corresponding TEMPO/acetate products (Scheme $4)$. Furthermore, different functional groups such as protected alcohol (3e) (Scheme 4), halide (3f) (Scheme 4) or ester (3g) (Scheme 4) were examined, all with very good results. For all of the examples above only the syn diastereoisomer was observed by NMR spectroscopy. However, when an alkene sub- 
strate containing a carboxylic acid was tested, $\delta$-lactone $\mathbf{3 h}$ derived from intramolecular attack was obtained in excellent yield. In this case the anti-isomer was formed selectively; this change in stereochemistry is due to the intramolecular nature of the nucleophile and supports our mechanistic proposal (see attack of a nucleophile from within the $\mathrm{R}^{1}$ group in $\mathbf{V}$, Scheme $2)^{24}$ Different carboxylic acid nucleophiles can be used; thus allyl TMS substrate in combination with propionic acid gives 3i with good results. Alternatively adding an aromatic acid or pivalic acid offers the possibility of accessing syn 1,2-diols $\mathbf{3 j}$ and $\mathbf{3 k}$ with diverse protecting groups.

Scheme 4. Scope of the metal-free dioxygenation with carboxylic acid incorporation.

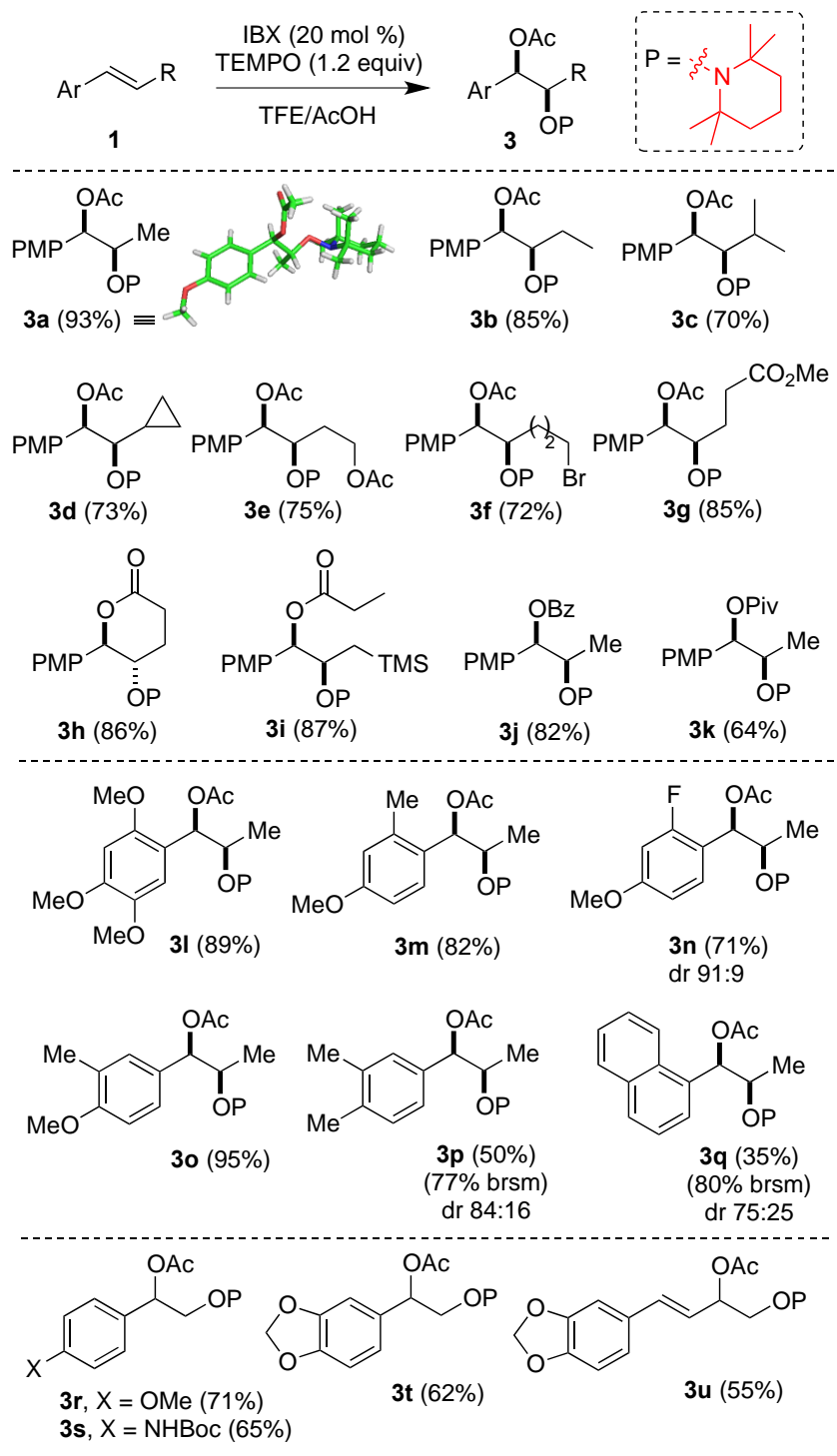

Moving to the aromatic ring we examined electron rich examples such as E-asarone (3l) (Scheme 4), 2-methyl (3m) (Scheme 4) or 3-methyl (3o) derivatives (Scheme 4) as well as those bearing other substituents, for example 2-fluoro (3n) (Scheme 4). The aryl olefin is not limited to $p$-methoxy styrenes and the method also works with less electron-donating substituents, for example 3,4-dimethyl (3p) or naphthalene substituents (3q) (Scheme 4), however in these cases the yield and diastereocontrol are compromised. Moreover, a representative group of terminal styrenes 3r-t (Scheme 4) and a diene 3u (Scheme 4) were tested with the latter example showing regioselectivity in favour of oxidation of the terminal olefin. Although the reaction scope is quite broad, the olefin must remain electron-rich for the reaction to proceed. In Scheme 4, the syn relative stereochemistry was proven using the X-Ray crystal structure of $\mathbf{3 a}$, $^{23}$ and the remaining compounds were assigned by analogy or by correlation of derivatives to compounds that are known in the literature, vide infra.

Finally in order to demonstrate the utility of this new method we addressed the selective deprotection of both hydroxy groups. Firstly, treating acetates 3a-d with $\mathrm{K}_{2} \mathrm{CO}_{3}$ in $\mathrm{MeOH}$ gave mono-protected alcohols 4a-d in excellent yields (Scheme 5a). In order to deprotect the OTEMP functionality, hexafluoroisopropyl ether $\mathbf{2 a}$ and esters $\mathbf{3 a}$ and $\mathbf{3 j}$ were subjected to reductive cleavage under standard conditions using $\mathrm{Zn} / \mathrm{AcOH}$ to form alcohols $\mathbf{5 a - c}$ in very good yields (Scheme 5a). Note that when acetate 3a was used alcohol $\mathbf{5 c}$ was obtained together with the acetate migration product (not shown, see supporting information). Fully deprotected syn 1,2-diols 6a-d were prepared by reaction of a mono-deprotected product under the complementary set of conditions (see $4 \mathbf{a}-\mathbf{d} \rightarrow \mathbf{6 a - d}$ via $\mathrm{N}-\mathrm{O}$ bond cleavage and $\mathbf{5} \mathbf{c} \rightarrow \mathbf{6 a}$ by acetate cleavage).

\section{Scheme 5. Functionalization of the OTEMP adducts.}

a) Selective deprotection

$$
\text { (2) }
$$

b) Deprotection and transesterification

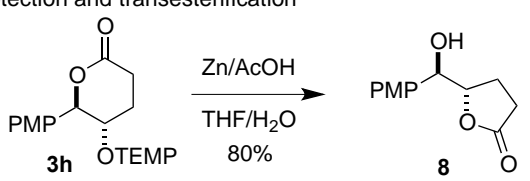

c) Cleavage of PMP group

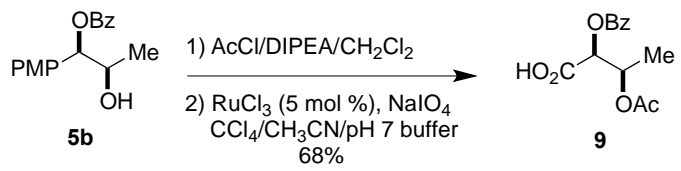

d) Heterocycle synthesis

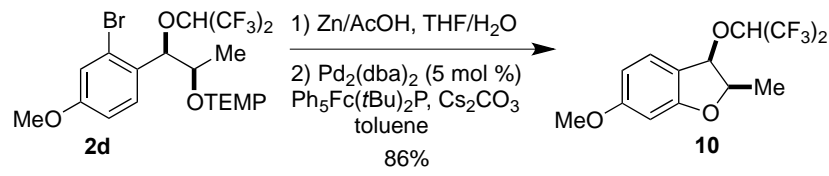

The spectroscopic data for syn 1,2-diols $\mathbf{6 a / 6 c}$ was in agreement with that previously reported in the literature, and the ${ }^{1} \mathrm{H} /{ }^{13} \mathrm{C}$ NMR spectroscopic data of diols $6 \mathbf{b}$ and $6 \mathbf{d}$ 
matched that of authentic syn diols made by $\mathrm{OsO}_{4}$ catalysed dihydroxylation of the corresponding $E$-alkene (see supporting information). In addition, ketones $\mathbf{7 a - b}$ were obtained by oxidative cleavage of the N-O bond with $m$-CPBA from $2 \mathbf{e}$ or $3 \mathbf{a}$ (Scheme $5 a) .{ }^{25}$ The deprotection of lactone $\mathbf{3 h}$ under reductive conditions resulted in the formation of transesterified product $\mathbf{8}$ in good yield and the NMR data for this anti-compound matched that in the literature (Scheme 5b). Alternatively the electron rich aromatic PMP ring can be oxidized to a carboxylic acid using catalytic $\mathrm{RuCl}_{3}{ }^{26}$ forming orthogonally protected $\alpha, \beta$-dihydroxy acids $\mathbf{9}$. Deprotection of $\mathbf{2 d}$ via reductive $\mathrm{N}-\mathrm{O}$ bond cleavage and subsequent $\mathrm{Pd}$ catalysed intramolecular $O$-arylation furnished dihydrobenzofuran $\mathbf{1 0}$ (Scheme 5d). Furthermore, nOe experiments on $\mathbf{1 0}$ supported its cisstereochemistry and therefore the syn stereochemistry of $\mathbf{2 d}$.

A new method for the syn-selective metal-free dioxygenation of electron rich olefins in fluorinated solvents has been presented; the procedure involves a proposed reaction with an in-situ generated oxoammonium cation, followed by the addition of a suitable nucleophile.

\section{ASSOCIATED CONTENT}

\section{Supporting Information}

The Supporting Information is available free of charge on the ACS Publications website.

Full experimental details, copies of spectral data (PDF) and crystallographic data (CIF)

\section{AUTHOR INFORMATION}

\section{Corresponding Author}

* E-mail: timothy.donohoe@chem.ox.ac.uk.

$\S$ Author to whom correspondence regarding the X-ray structures should be addressed.

Notes

The authors declare no competing financial interest.

\section{ACKNOWLEDGMENT}

We thank the European Union and the European Commission for financial support (IC): the research leading to these results has received funding from the People Programme (Marie Curie Actions) of the European Union's Seventh Framework Programme (FP7/2007-2013). RCB thanks FAPESP (Award No. 2014/165169) for funding. We thank W. Akhtar and Dr. A. L. Thompson (University of Oxford) for assistance with the single crystal X-ray diffraction studies.

\section{REFERENCES}

(1) Zaitsev, A. B.; Adolfsson, H. Synthesis 2006, 1725-1756.

(2) For metal-free dihydroxylation see: (a) Rawling, M. J.; Tomkinson, N. C. O. Org. Biomol. Chem. 2013, 11, 1434-1440. (b) Bataille, C. J. R.; Donohoe, T. J. Chem. Soc. Rev. 2011, 40, 114-128.

(3) Griffith, J. C.; Jones, K. M.; Picon, S.; Rawling, M. J.; Kariuki, B. M.; Campbell, M.; Tomkinson, N. C. O. J. Am. Chem. Soc. 2010, 132, 14409-14411.

(4) Schmidt, V. A.; Alexanian, E. J. Angew. Chem. Int. Ed. 2010, 49, 4491-4494.

(5) (a) Emmanuvel, L.; Shaikh, T. M. A.; Sudalai, A. Org. Lett. 2005, 7, 5071-5074. (b) Çelik, M.; Alp, C.; Coşkun, B.; Gültekin, M. S.; Balci, M. Tetrahedron Lett. 2006, 47, 3659-3663. (c) Zhong, W.; Yang, J.; Meng, X.; Li, Z. J. Org. Chem. 2011, 76, 9997-10004. (d) Zhong, W.; Liu, S.; Yang, J.; Meng, X.; Li, Z. Org. Lett. 2012, 14, 3336-3339. (e) Bekkaye, M.; Su, Y.; Masson, G. Eur. J. Org. Chem. 2013, 3978-3982.
(6) (a) Fujita, M.; Wakita M.; Sugimura, T. Chem. Commun. 2011, 47, 3983-3985

(7) (b) Fujita, M.; Yoshida, Y.; Miyata, K.; Wakisaka, A.; Sugimura, T. Angew. Chem. Int. Ed. 2010, 49, 7068-7071.

(8) Haubenreisser, S.; Wöste, T. H.; Martínez, C.; Ishihara, K.; Muñiz, K. Angew. Chem. Int. Ed. 2016, 55, 413-417.

(9) For a review: Vogler, T.; Studer, A. Synthesis 2008, 1979-1993.

(10) Li, Y.; Hartmann, M.; Daniliuc, C. G.; Studer, A. Chem. Commun. 2015, 51, 5706-5709.

(11) Zhang, B.; Studer, A. Org. Lett. 2013, 15, 4548-4551.

(12) Hartmann, M.; Li, Y.; Studer, A. J. Am. Chem. Soc. 2012, 134, 16516-16519.

(13) Li, Y.; Studer, A. Angew. Chem. Int. Ed. 2012, 51, 8221-8224.

(14) See: Xia, X.-F.; Zhu, S.-L.; Niu, Y.-N.; Zhang, D.; Liu, X.; Wang, H. Tetrahedron 2016, 72, 3068-3072.

(15) (a) Colomer, I.; Coura Barcelos, R.; Donohoe, T. J. Angew. Chem. Int. Ed. 2016, 55, 4748-4752. (b) Colomer, I.; BatchellorMcAulley, C.; Odell, B.; Donohoe, T. J.; Compton, R. G. J. Am. Chem. Soc. 2016, 138, 8855-8861.

(16) We have not observed oxidation of the fluorinated solvent, although TFE can be oxidized by oxoammonium salts or DMP: (a) Linderman R. J.; Graves, D. M.; Tetrahedron Lett. 1987, 28, 42594262. (b) Ignatowska, J.; Shyshkov, O.; Zipplies, T.; Hintzer, K.; Röschenthaler, G.-V. J. Fluorine Chem. 2012, 141, 35-40.

(17) (a) de Nooy, A. E. J.; Besemer, A. C.; van Bekkum, H. Tetrahedron 1995, 51, 8023-8032. (b) Ma, Z.; Bobbitt, J. M. J. Org. Chem. 1991, 56, 6110-6114.

(18) (a) Kelly, C. B.; Lambert, K. M.; Mercadante, M. A.; Ovian, J. M.; Bailey, W. F.; Leadbeater, N. E. Angew. Chem. Int. Ed. 2015, 54, 4241-4245. (b) Kelly, C. B.; Mercadante, M. A.; Wiles, R. J.; Leadbeater, N. E. Org. Lett. 2013, 15, 2222-2225.

(19) For a similar discrete benzylic carbocation see: Mohan, R. S.; Whalen, D. L. J. Org. Chem. 1993, 58, 2663-2269.

(20) See: (a) Pradhan, P. P.; Bobbitt, J. M.; Bailey, W. F. Org. Lett. 2006, $8,5485-5487$. We are aware of little precedent for the reaction of oxoammonium cations with electron rich olefins: (b) Takata, T.; Tsujino, Y.; Nakanishi, S.; Nakamura, K.; Yoshida, E.; Endo, T. Chem. Lett. 1999, 9, 937-938.

(21) We propose that IBX (Iodine-V) can oxidize TEMPO-H to oxoamonioum cation IV generating a hypervalent iodine (III) specie that could engage in a second TEMPO-H oxidation process, therefore requiring only $20 \mathrm{~mol} \%$ of IBX for the best efficiency. The ability of hypervalent iodine (III) to oxide TEMPO-H to its oxoamonium cation is well established: De Mico, A.; Margarita, R.; Parlanti, L.; Vescovi, A.; Piancatelli, G. J. Org. Chem. 1997, 62, 6974-6977.

(22) For reviews of HFIP see: (a) Bégué, J.-P.; Bonnet-Delpon, D.; Crousse, B. Synlett 2004, 18-29. (b) Shuklov, I. A.; Dubrovina, N. V.; Börner, A. Synthesis 2007, 2925-2943.

(23) Low temperature single crystal X-ray diffraction data were collected using a (Rigaku) Oxford Diffraction SuperNova diffractometer, (a) Cosier, J.; Glazer, A. M. J. Appl. Cryst. 1986, 19, 105-107. Raw frame data were reduced using CrysAlisPro and the structures were solved using 'Superflip' before refinement with CRYSTALS as per the SI (CIF). (b) Palatinus, L.; Chapuis, G. $J$. Appl. Cryst. 2007, 40, 786-790. (c) P. Parois, R.I. Cooper and A.L. Thompson Chem. Cent. J. 2015, 9, 30. (d) Cooper, R. I.; Thompson, A. L.; Watkin, D. J. J. Appl. Cryst. 2010, 43, 11001107. Full refinement details are given in the Supporting Information (CIF); Crystallographic data have been deposited with the Cambridge Crystallographic Data Centre (CCDC 1498189-90) and can be obtained via www. ccdc.cam.ac.uk/data_request/cif..

(24) See the Supporting Information for a complete NMR study of $\delta$-lactone $\mathbf{3 h}$.

(25) Inokuchi, T.; Kawafuchi, H. Tetrahedron 2004, 60, 1196911975.

(26) Carlsen, P. H. J.; Katsuki, T.; Martin, V. S.; Sharpless, K. B. J. Org. Chem. 1981, 46, 3936-3938. 
